\title{
Construction of recombinant adenoviruses carrying the optimal shRNA template against goat PTHrP and successful suppression of PTHrP expression in mammary epithelial cells
}

\author{
H.L. Zheng, X.M. Liu, Q. Yu, R.F. Xing, Z.Y. Yang and J. Luo \\ College of Animal Science and Technology, \\ Northwest Agriculture and Forestry University, \\ Key Laboratory of Molecular Biology for Agriculture, \\ Shaanxi Province, Yangling, China \\ Corresponding authors: J. Luo / H.L. Zheng \\ E-mail: luojun1@yahoo.com / zheng.huiling@yahoo.com
}

Genet. Mol. Res. 12 (3): 3363-3374 (2013)

Received September 13, 2012

Accepted December 21, 2012

Published September 4, 2013

DOI http://dx.doi.org/10.4238/2013.September.4.2

\begin{abstract}
Parathyroid hormone-related protein $(P T H r P)$ is a protein member of the parathyroid hormone family that regulates the dynamic balance between blood and bone calcium during lactation. However, the mechanism of its regulation is not very clear. In order to establish a framework for further functional studies of the $\mathrm{PTHrP}$ gene in goat mammary gland epithelial cells during the lactation period, $P T H r P$ cDNA was isolated from Xinong Saanen dairy goats. Its coding sequence is $534 \mathrm{bp}$ in size. We also designed a short hairpin RNA (shRNA) to efficiently inhibit $P T H r P$ expression and constructed recombinant adenoviruses carrying a template encoding this shRNA (AD-PTHrP-322) using the Block-iT shRNA interference system. Finally, the inhibition of PTHrP expression by the recombinant adenoviruses was confirmed by quantitative reverse transcriptasepolymerase chain reaction (qRT-PCR) and Western blotting. qRT-PCR results showed that the expression of $P T H r P$ mRNA in mammary
\end{abstract}


epithelial cells was downregulated by $29.2,68.1$, and $82.6 \% 24,48$, and $72 \mathrm{~h}$ after the cells were infected with AD-PTHrP-322, respectively. Western blotting also showed that the expression of $P T H r P$ was reduced in a time-dependent manner. These results suggest that AD-PTHrP-322 significantly inhibits the expression of PTHrP.

Key words: PTHrP; RNA interference; Recombinant adenovirus

\section{INTRODUCTION}

Parathyroid hormone-related protein $(\mathrm{PTHr} P)$ was originally discovered as a tumor product that causes humoral hypocalcemia of malignancy (Philbrick et al., 1996). It is also widely expressed in fetal and adult tissues and is a key regulator of cellular calcium transport and smooth muscle cell contractility, as well as a crucial factor in cell proliferation, development, and differentiation (Kremer et al., 2011). PTHrP is involved in a wide range of developmental and physiological processes. It is secreted into the circulation by the lactating mammary gland and it regulates the concentration of serum calcium during lactation (VanHouten et al., 2003; Lozano et al., 2009; Hildreth et al., 2010). It stimulates the secretion of calcium, phosphate, and magnesium in milk and it might foster the development of the mammary glands (Barlet et al., 1992; Onda et al., 2010). PTHrP is necessary for the maintenance of the mammary bud. Loss of $P T H r P$ or its receptor causes the mammary bud cell to revert to an epidermal fate. In lactation, it may regulate the mobilization of binding of the ligand with the calcium-sensing receptor, the transfer of calcium to the milk, as well as placental transfer of calcium (Hens and Wysolmerski, 2005; Hens et al., 2007). This evidence suggests that $\mathrm{PTHr} P$ plays an important role in regulating the dynamic balance between blood calcium and bone calcium during the lactation period. However, the mechanism of the regulation of this gene is not currently understood.

RNA interference (RNAi) is a normal phenomenon in eukaryotic cells that protects the cells from viruses and inhibits the expression of specific genes through the action of small interfering RNAs (siRNAs) (Gaither and Iourgenko, 2007). RNAi was also recognized as a major means of gene regulation and a powerful tool for functional gene analysis in mammalian cells (Barik, 2005; Huai and Ma, 2009).

In order to understand the mechanism by which $P T H r P$ regulates the concentration of serum calcium during lactation, we constructed recombinant adenoviruses carrying the template for a small hairpin RNA (shRNA) that efficiently reduces $P T H r P$ expression. The sequence of the shRNA was designed based on the sequence of the PTHrP-coding sequence (CDS) of goat that we cloned. The shRNA was cloned into the Block-iT shRNA interference system to construct recombinant adenovirus vectors, which were co-transfected with Lipofectamine 2000 (Invitrogen, USA) into human embryonic kidney (HEK)-293 cells for packing and amplifying recombinant adenoviruses. Fourth-generation recombinant adenoviruses with high titer were used to infect mammary epithelial cells. PTHrP expression in the mammary epithelial cells infected with the recombinant adenoviruses was measured by quantitative reverse-transcriptase-polymerase chain reaction (qRT-PCR) and Western blotting, both of which confirmed the successful construction of recombinant adenoviruses carrying an efficient shRNA targeting goat PTHrP. 


\section{MATERIAL AND METHODS}

\section{Cloning of the goat PTHrP gene}

Total RNA extracted from goat mammary gland using Trizol reagent (Tektronix, China) was used for cDNA first-strand synthesis using the SuperScript II First-Strand Synthesis System (Tiangen Biotech Co., Ltd., China). This cDNA was used as the template for qRT-PCR with the following PCR primers to amplify the PTHrP transcript: $P T H r P-B g l I I-F$ : 5'-GAAGATCTGCGAGAGGATACGATGCT-3' and PTHrP-XhoI-R: 5'-CCGCTCGAGTGAA GGTCTCTGCTGAAAAAT- 3 '. These primers were designed based on the sequence of the conserved regions of bovine PTHrP (GenBank accession No. BC149411.1) with the Primer 5.0 software (Premier, Canada). PCR cycling conditions were $95^{\circ} \mathrm{C}$ for $30 \mathrm{~s}, 48^{\circ} \mathrm{C}$ for $1 \mathrm{~min}, 72^{\circ} \mathrm{C}$ for $1 \mathrm{~min}$ for 35 cycles. Next, the PCR products were purified by agarose gel electrophoresis and directly sub-cloned into the pMD-19T vector (TaKaRa, China). The ligated products were transformed into TOP10 Escherichia coli competent cells (Tiangen Biotech Co., Ltd.) using the calcium chloride method. Positive colonies were selected by blue-white spot screening and resistances of ampicillin and plasmid DNA was extracted using the TIANpure Midi Plasmid Kit (Tiangen Biotech Co., Ltd.). The recombinant plasmid was screened by $B g l \mathrm{II} / \mathrm{XhoI}$ restriction enzyme digestion and further confirmed by sequencing.

\section{Design and synthesis of shRNA sequence targeting goat PTHrP mRNA}

Based on the principles of siRNA design and the full-length CDS of the goat $P T H r P$ gene, 3 pairs of complementary single-stranded DNA oligonucleotides were designed using an online software (http: //www.promega.com/siRNADesigner/program/default.asp). Two pairs of oligonucleotides targeted 2 different sites of PTHrP mRNA. The third oligonucleotide pair was designed as a negative control. The oligonucleotides were synthesized by the Shanghai Biological Engineering Company (China).

\section{Construction of pENTR/CMV-GFP/U6-shRNA}

The 3 pairs of single-stranded DNAs were diluted to $10 \mu \mathrm{M}$ for renaturation. The renaturation conditions were $95^{\circ} \mathrm{C}$ for $10 \mathrm{~min}, 90^{\circ} \mathrm{C}$ for $10 \mathrm{~s}$, and then a gradient step where the temperature decreased by $5^{\circ} \mathrm{C}$ every $10 \mathrm{~s}$ until it reached $50^{\circ} \mathrm{C}$. The 3 resulting double-stranded DNAs with sticky ends are referred to as PTHrP-shRNA-322, PTHrT-shRNA-357, and PTHrT-shRNA-NC.

The Block-iT shRNA interference system (including the adenovirus shuttle vector pENTR/CMV-GFP/U6 and the adenovirus backbone vector pAD/PL-DEST) was used in this study. Plasmid pENTR/CMV-GFP/U6 was double-digested with BamHI and XhoI restriction endonucleases (TaKaRa). The linearized large fragment of pENTR/CMV-GFP/U6 was extracted from an agarose gel after digestion, and then incubated with PTHrT-shRNA in a 3:1 ratio with T4 DNA ligase at $4^{\circ} \mathrm{C}$ overnight. Ligated products were transformed into competent TOP $10 \mathrm{E}$. coli cells by the thermal excitation method. Colonies were grown on Luria-Bertani (LB) $(0.5 \%$ yeast extract, $1 \%$ tryptone, $1 \% \mathrm{NaCl}$, and $1.2 \%$ agar) plates containing kanamycin. They were picked and propagated for plasmid DNA preparation. Colonies were screened by double-digestion and successful cloning was further verified by sequencing. The positive clones are referred to as 
pENTR/CMV-GFP/U6-322, pENTR/CMV-GFP/U6-357, and pENTR/CMV-GFP/U6-NC.

\section{Identification of the optimal shRNA sequence}

In order to identify the optimal shRNA template with the highest knockdown efficiency, $\mathrm{pENTR/CMV-GFP/U6-shRNA} \mathrm{was} \mathrm{transfected} \mathrm{alone} \mathrm{or} \mathrm{co-transfected} \mathrm{with} \mathrm{pADTrack-CMV-}$ PTHrP, which can overexpress PTHrP of goat and constructed by our laboratory into HEK-293 cells at $70-80 \%$ confluence with Lipofectamine 2000 (Invitrogen). Then, the transfected HEK293 cells were cultured in Dulbecco's modified Eagle's medium (DMEM) supplemented with $10 \%$ fetal bovine serum (FBS) and incubated at $37^{\circ} \mathrm{C}$ with $5 \% \mathrm{CO}_{2}$. At 24 -h post-transfection, the GFP expression levels were determined using fluorescence microscopy. At $48 \mathrm{~h}$ after transfection, relative PTHrP expression levels were determined by Western blotting. Briefly, the transfected cells were centrifuged at $900 \mathrm{~g}$ for $5 \mathrm{~min}$. After removing the supernatant, the cells were lysed by Radio Immunoprecipitation Assay (Invitrogen), followed by centrifugation, and the supernatant was collected to quantify the concentration of total protein by the bicinchoninic acid (Thermo Scientific, China) method. An aliquot of the lysate was loaded onto a 10\% sodium dodecyl sulfate-polyacrylamide gel. Following electrophoresis, the protein was transferred to a polyvinyl difluoride membrane (Yeli Bioscience Co., Ltd., China). Next, the polyvinyl difluoride membrane was blocked with 5\% skim milk for $2 \mathrm{~h}$ at room temperature and then washed with Tris-buffered saline-Tween ( $1 \mathrm{M}$ Tris-HCl, $0.88 \% \mathrm{NaCl}$, and $0.05 \%$ Tween 20, pH 7.4) 3 times. The membrane was then co-incubated with a $P T H r P$ rabbit anti-goat polyclonal antibody that was made by our laboratory $\left(1: 1000\right.$ dilution) overnight at $4^{\circ} \mathrm{C}$. After the membrane was rinsed in Tris-buffered saline-Tween to remove unbound primary antibody, a specific secondary antibody (1:5000 horseradish peroxidase-conjugated goat-anti-rabbit IgG; Bioss, China) was added and incubated at $37^{\circ} \mathrm{C}$ for $1 \mathrm{~h}$. Western blots were visualized with the enhanced chemiluminescence Western blotting detection reagents (Tiangen Biotech Co., Ltd.). The optimal shRNA template thus identified was used for further study.

\section{Construction of pAD/PL-DEST/CMV-GFP/U6-shRNA}

A recombination reaction between $\mathrm{pENTR/CMV-GFP/U6-shRNA} \mathrm{and} \mathrm{the} \mathrm{adenovi-}$ rus backbone vector pAD/PL-DEST was performed overnight using LR Clonase (Invitrogen) at $25^{\circ} \mathrm{C}$. Reaction products were transformed into TOP10 E. coli cells (Tiangen Biotech Co., Ltd.). Five microliters of transformed cells was spread onto solid LB medium containing ampicillin and incubated at $37^{\circ} \mathrm{C}$ overnight for selection. Single clones were picked and inoculated in LB liquid medium and propagated at $37^{\circ} \mathrm{C}$ for $10 \mathrm{~h}$. Some of the inoculum was streaked on LB-chloramphenicol plates. Clones that could not grow on LB-chloramphenicol plates were defined preliminarily as positive clones. We then used agarose gel electrophoresis and sequencing to further verify the sequence of the clones. The positive clones are referred to as pAD/PL-DEST/CMV-GFP/U6-322 and pAD/PL-DEST/CMV-GFP/U6-NC.

\section{Generation of recombinant adenoviruses carrying the optimal shRNA template against goat $\mathrm{PTHrP}$}

The recombinant adenoviral plasmid pAD/PL-DEST/CMV-GFP/U6-shRNA-322 was 
linearized by $P a c \mathrm{I}$ restriction enzyme digestion (Thermo Scientific) and transfected into HEK293 cells at $60-70 \%$ confluence with Lipofectamine 2000 to package recombinant adenoviruses. At 3 days post-transfection, relative GFP expression levels were determined by fluorescence microscopy. When about $50 \%$ of the cells began to show a cytopathic phenotype, the first generation of adenovirus was collected by 3 freeze-thaw cycles using liquid nitrogen, followed by centrifugation at $900 \mathrm{~g}$ for $10 \mathrm{~min}$. The virus-containing supernatant was collected and used to infect HEK293 cells for the amplification of the recombinant adenovirus. The infection cycle was repeated 4 times to generate high titer adenovirus. The titer was determined by $50 \%$ tissue culture infective dose $\left(\mathrm{TCID}_{50}\right)$ assays and the recombinant adenoviruses were stored at $-80^{\circ} \mathrm{C}$ as viral stock.

\section{Evaluation of $P T H r P$ gene silencing by recombinant adenoviruses carrying the optimal shRNA template against goat $\mathrm{PTHrP}$}

PTHrP expression in the transfected mammary epithelial cells was measured by qRTPCR and Western blotting. Primary goat mammary epithelial cells isolated from healthy goat mammary gland and cultured by our laboratory in advance $\left(5 \times 10^{5}\right.$ cells/well) were seeded on 12-well plates in DMEM supplemented with $10 \% \mathrm{FBS}$ and incubated overnight at $37^{\circ} \mathrm{C}$ with $5 \% \mathrm{CO}_{2}$. The medium was replaced before transfection. Mammary epithelial cells were transfected at $80-90 \%$ confluence, with 200 multiplicity of infection recombinant adenovirus carrying anti-goat $P T H r P$ shRNA. The mammary epithelial cells were harvested at 24,48 , and $72 \mathrm{~h}$ post-transfection.

Total RNA was isolated from the mammary epithelial cells and reverse transcribed into cDNA. qRT-PCR primers for the goat $P T H r P$ gene were designed based on the $P T H r P$ gene sequence (GenBank accession No. JQ409458) with the Primer 5.0 software. $\beta$-actin was used as the reference gene. The respective forward and reverse primers for $\beta$-actin were as follows: 5'-ACTGAGGCCATGATTAAG-3'，5'-GCTATCAATCTGTCAATCC-3'. qRT-PCR was performed with the SYBR Premix Ex Taq ${ }^{\text {TM }}$ (Perfect Real-Time) kit (TaKaRa) on the ABI StepOnePlus fluorescence quantitative PCR instrument (BIO-RAD, USA). qRT-PCR was performed in a $20-\mu \mathrm{L}$ reaction mixture containing $10 \mu \mathrm{L} 2 \mathrm{X}$ SYBR Green premix, $0.8 \mu \mathrm{L} 20 \mathrm{pM}$ each primer, 2 $\mu \mathrm{L}$ template $\mathrm{cDNA}$, and $6.4 \mu \mathrm{L}$ diethylpyrocarbonate DEPC water. The amplification conditions were $95^{\circ} \mathrm{C}$ for $3 \mathrm{~min}$, and 40 cycles of $95^{\circ} \mathrm{C}$ for $10 \mathrm{~s}, 56^{\circ} \mathrm{C}$ for $20 \mathrm{~s}$, and $72^{\circ} \mathrm{C}$ for $20 \mathrm{~s}$.

Total protein for Western blotting analysis was extracted from mammary epithelial cells as described above.

\section{Statistical analysis}

Every experiment was repeated independently at least 3 times. The significance of differences was assessed by one-way analysis of variance using the SPSS 13.0 software (SPSS, USA). $\mathrm{P}<0.05$ was considered to be significant.

\section{RESULTS}

\section{Cloning of the goat PTHrP gene}

We successfully cloned the complete CDS of the goat PTHrP gene. It is $534 \mathrm{bp}$ long 
(Figure 1) and its sequence can be accessed through GenBank (GU573787). The PTHrP cDNA sequence is predicted to encode a 177 -amino acid protein with a relative molecular weight of $20.3 \mathrm{kDa}$.

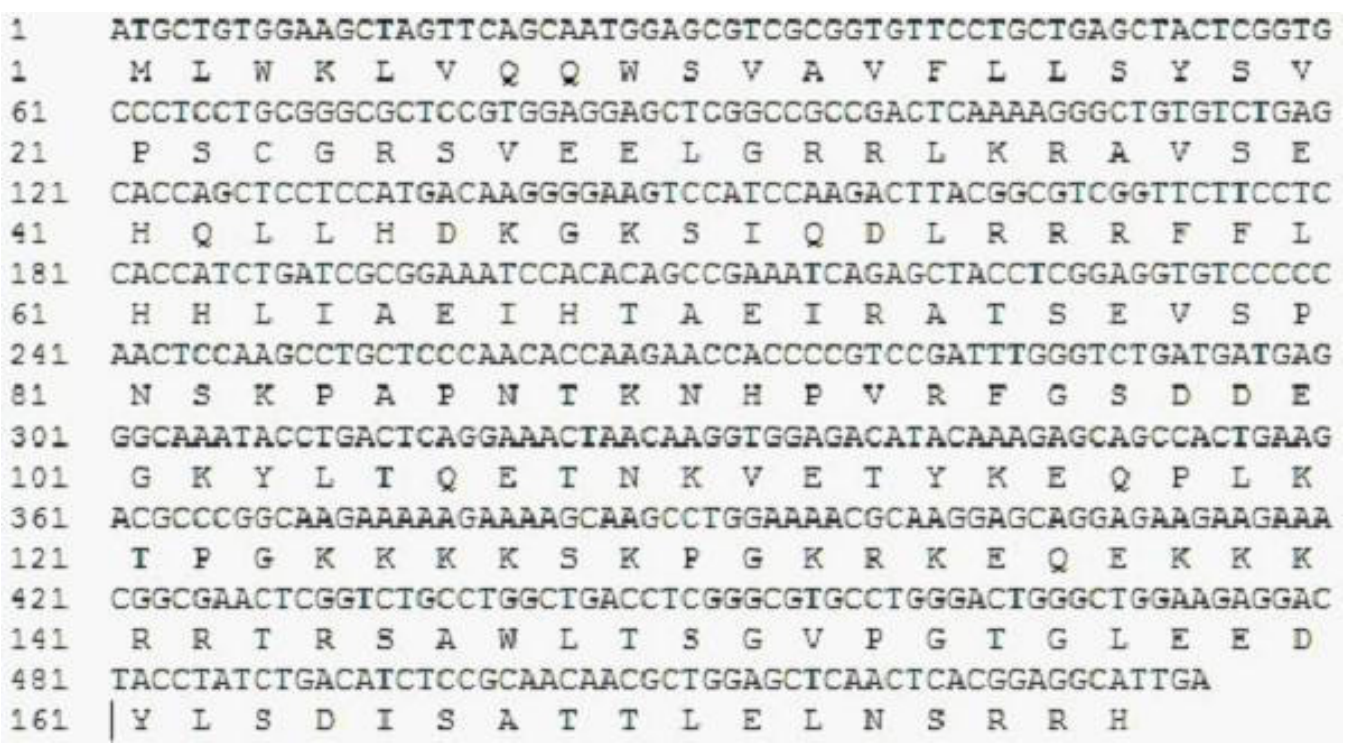

Figure 1. cDNA and its deduced predicted protein sequence of the goat $P T H r P$ gene. Nucleotides and amino acids are numbered to the left of the sequence.

\section{shRNA-based targeting of the goat PTHrP mRNA}

Six single-stranded oligonucleotide sequences targeting the goat $P T H r P$ mRNA were synthesized by Shanghai Biological Engineering Company and they are shown in Table 1. The first 2 pairs of oligonucleotides targeted 2 different sites of the PTHrP mRNA whereas the remaining pair was a negative control.

Table 1. shRNA sequence targeting $P T H r P$ of goat.

\begin{tabular}{ll}
\hline Name & Sequence $\left(5^{\prime}-3^{\prime}\right)$ \\
\hline shRNA-322 sense & GATCCCTAACAAGGTGGAGACATATTCAAGAGATATGTCTCCACCTTGTTAGTTTTTTC \\
shRNA-322 antisense & TCGAGAAAAAACTAACAAGGTGGAGACATATCTCTTGAATATGTCTCCACCTTGTTAGG \\
shRNA-357 sense & GATCCGAAGACGCCCGGCAAGAAATTCAAGAGATTTCTTGCCGGGCGTCTTCTTTTTTC \\
shRNA-357 antisense & TCGAGAAAAAAGAAGACGCCCGGCAAGAAATCTCTTGAATTTCTTGCCGGGCGTCTTCG \\
shRNA-NC sense & GATCCTTCTCCGAACGTGTCACGTTTCAAGAGAACGTGACACGTTCGGAGAATTTTTC \\
shRNA-NC antisense & TCGAGAAAAAATTCTCCGAACGTGTCACGTTCTCTTGAAACGTGACACGTTCGGAGAAG \\
\hline
\end{tabular}

\section{Construction of pENTR/CMV-GFP/U6-shRNA}

The pENTR/CMV-GFP/U6 plasmid was double-digested with the BamHI and XhoI restriction enzymes. The $\sim 4.6-\mathrm{kb}$ digestion product can be seen in Figure $2 \mathrm{~A}$. The recombinant 
plasmid pENTR/CMV-GFP/U6-shRNA was also double-digested with BamHI and XhoI. The circular plasmid was cleaved into a 4.6-kb pENTR/CMV-GFP/U6 DNA fragment and a 59-bp $P T H r P$ gene fragment (Figure 2B). Further sequencing tests confirmed the successful cloning of pENTR/CMV-GFP/U6-322, pENTR/CMV-GFP/U6-357, and pENTR/CMV-GFP/U6-NC.
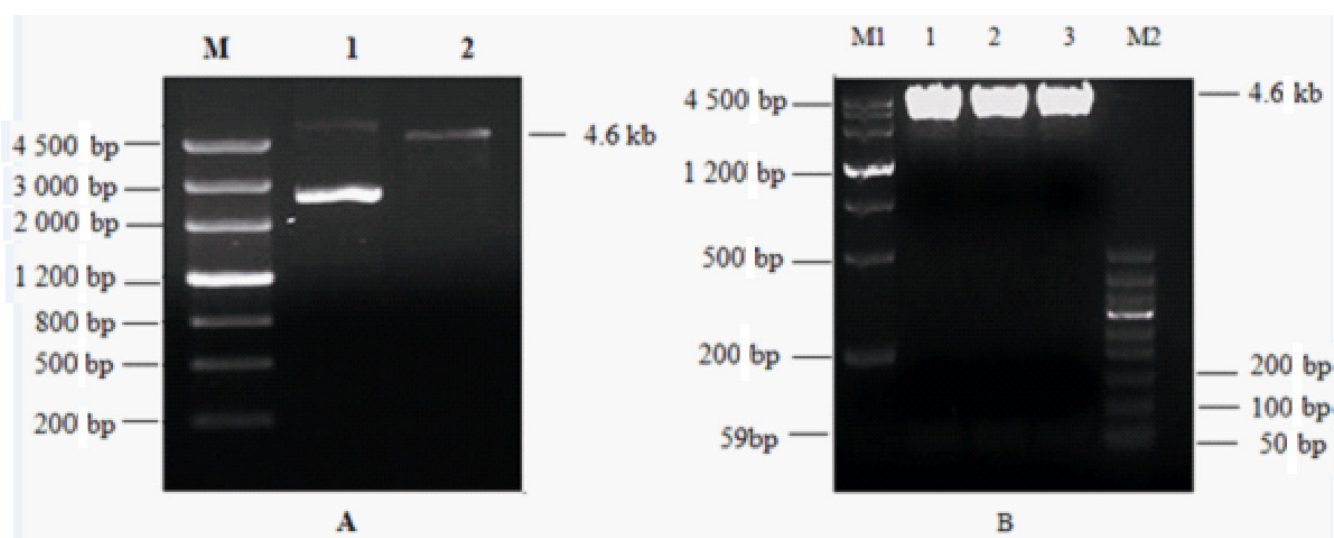

Figure 2. A. Agarose gel electrophoresis of pENTR/CMV-GFP/U6 digested by BamHI and XhoI. Lane $1=\mathrm{pENTR} /$ CMV-GFP/U6; lane 2 = double-digested products; lane $M=$ marker b. B. Agarose gel electrophoresis of pENTR/ CMV-GFP/U6-shRNA plasmid digested with BamHI and XhoI. Lanes 1 to $3=$ pENTR/CMV-GFP/U6-322, -357, and $-\mathrm{NC}$, respectively; lane $M 1=$ marker b; lane $M 2=50$-bp ladder.

\section{Identification of the optimal shRNA sequence}

The PTHrP expression levels in HEK-293 cells transfected with the plasmids are shown by Western blotting in Figure 3. The PTHrP protein could not be detected in untransfected HEK-293 cells by Western blotting. However, it was detected in cells transfected with pADTrack-CMV-PTHrP. The Western blotting results indicated that both $\mathrm{pENTR/CMV-GFP/}$ U6-322 and pENTR/CMV-GFP/U6-357 had the ability to knockdown PTHrP gene expression, with the former showing greater gene knockdown. pENTR/CMV-GFP/U6-NC did not have any effect on PTHrP gene expression. These results suggested that $\mathrm{pENTR/CMV-GFP/}$ U6-322 could be used for constructing the recombinant virus and that $\mathrm{pENTR/CMV-GFP/U6-}$ $\mathrm{NC}$ could be used as negative control.

\section{Generation of recombinant adenoviruses carrying the optimal anti-goat $\mathrm{PTHrP}$ shRNA template}

The adenovirus backbone vector $\mathrm{pAD} / \mathrm{PL}-\mathrm{DEST}$ and the recombinant plasmid $\mathrm{pAD} /$ PL-DEST/CMV-GFP/U6-shRNA were separated by agarose gel electrophoresis (Figure 4). $\mathrm{pAD} / \mathrm{PL}-\mathrm{DEST}$ is about $35 \mathrm{~kb}$ and pAD/PL-DEST/CMV-GFP/U6-shRNA is about $40 \mathrm{~kb}$. The recombinant plasmid sequences were further verified by sequencing to confirm that we had successfully constructed pAD/PL-DEST/CMV-GFP/U6-322 and pAD/PL-DEST/CMV-GFP/ U6-NC (Figure 4). 


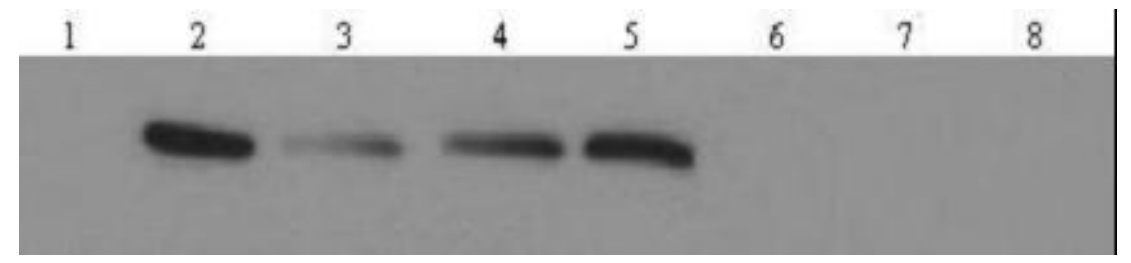

Figure 3. Detection of interference efficiency of shRNA in HEK-293 by Western blotting. Lane $1=$ blank control; lane 2 = transfected by pADTrack-CMV-PTHrP; lane 3 = co-transfected by pENTR/CMV-GFP/U6-322 and pADTrack-CMV-PTHrP; lane 4 = co-transfected by pENTR/CMV-GFP/U6-357 and pADTrack-CMV-PTHrP; lane $5=$ co-transfected by pENTR/CMV-GFP/U6-NC and pADTrack-CMV-PTHrP; lane $6=$ transfected by $\mathrm{pENTR/CMV-GFP/U6-322;} \mathrm{lane} 7=$ transfected by $\mathrm{pENTR/CMV-GFP/U6-357;} \mathrm{lane} 8=$ transfected by pENTR/ CMV-GFP/U6-NC.

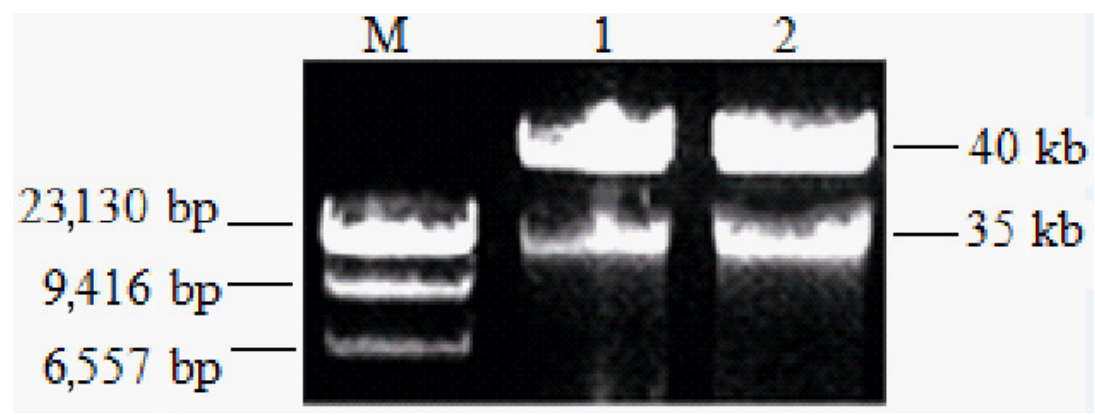

Figure 4. Agarose gel electrophoresis of pAD/PL-DEST/CMV-GFP/U6-shRNA. Lanes 1 and 2 = pAD/PL-DEST/ CMV-GFP/U6-322 and -NC, respectively; lane $M=\lambda \mathrm{DNA} /$ Hind.

pAD/PL-DEST/CMV-GFP/U6-322 and pAD/PL-DEST/CMV-GFP/U6-NC were linearized by $\mathrm{PacI}$ restriction enzyme digest and were transfected into HEK-293 cells for viral packaging. GFP expression levels were determined by fluorescence microscopy. At 3 days post-transfection, we saw weak green fluorescence. At 6 days, the relative fluorescence was greater and some cells began to show a cytopathic phenotype. On the 12 th day, most of the cells became round and floated in the medium because of decreased adhesion (Figure 5). The adenoviruses were collected before the cells ruptured, and then retransfected into HEK-293 cells 4 times to generate a high titer of adenovirus. We refer to the 4th generation of adenoviruses as AD-PTHrP-322 and AD-PTHrP-NC. The titers of the AD-PTHrP-322 and AD-PTHrP-NC adenoviruses reached $2.0 \times 10^{9}$ and $1.3 \times 10^{9}$ plaque-forming units/mL by TCID $_{50}$ assays, respectively. We also found that the optimum multiplicity of infection of AD-PTHrP-322 was 200 by infecting primary goat mammary gland epithelial cells.

\section{pAD/PL-DEST/CMV-GFP/U6-322 silences PTHrP gene expression in mammary epithelial cells}

Sense and antisense primers to amplify the goat $P T H r P$ transcript by qRT-PCR were designed based on the sequence of $P T H r P$ with the Primer 5.0 software (Table 2). 


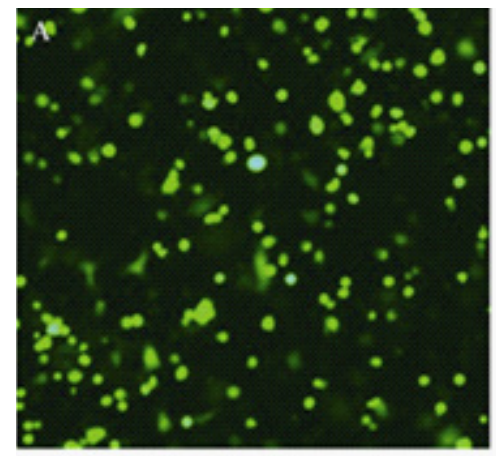

A

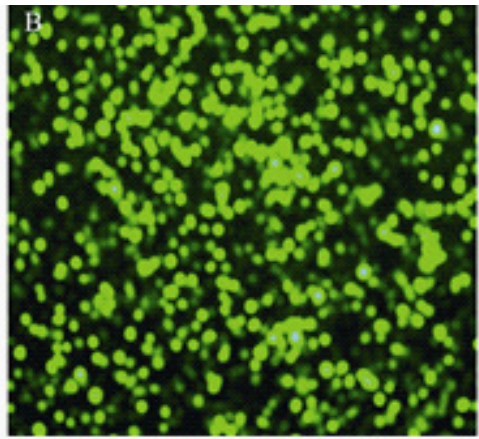

B

Figure 5. Expression of GFP in the process of adenovirus package 6 and 12 days under fluorescence microscopy. A. 6 days; B. 12 days.

Table 2. Primer sequences used for real-time PCR.

\begin{tabular}{|c|c|c|c|}
\hline Name & Sequences $\left(5^{\prime}-3^{\prime}\right)$ & $\mathrm{Ta}\left({ }^{\circ} \mathrm{C}\right)$ & Length (bp) \\
\hline $\begin{array}{l}P T H r P \text { sence } \\
P T H r P \text { antisence }\end{array}$ & $\begin{array}{l}\text { TTACGGCGTCGGTTCTTCCT } \\
\text { GCGTCTTCAGTGGCTGCTCT }\end{array}$ & 56 & 205 \\
\hline$\beta$-actin sence & TACGGCGTCGGTTCTTCCT & 56 & 142 \\
\hline$\beta$-actin antisence & СССТCATCATCAGACCCAAAT & & \\
\hline
\end{tabular}

The qRT-PCR results showed that $P T H r P$ mRNA expression was downregulated by $29.2,68.1$, and $82.6 \%$ in the mammary epithelial cells 24,48 , and $72 \mathrm{~h}$ after infection with AD-PTHrP-322, respectively ( $\mathrm{P}<0.05$ compared with expression in untransfected cells) (Figure 6). The PTHrP mRNA expression levels gradually reduced over time, suggesting that pAD/PL-DEST/CMV-GFP/U6-322 had the ability to interfere with $P T H r P$ gene expression.

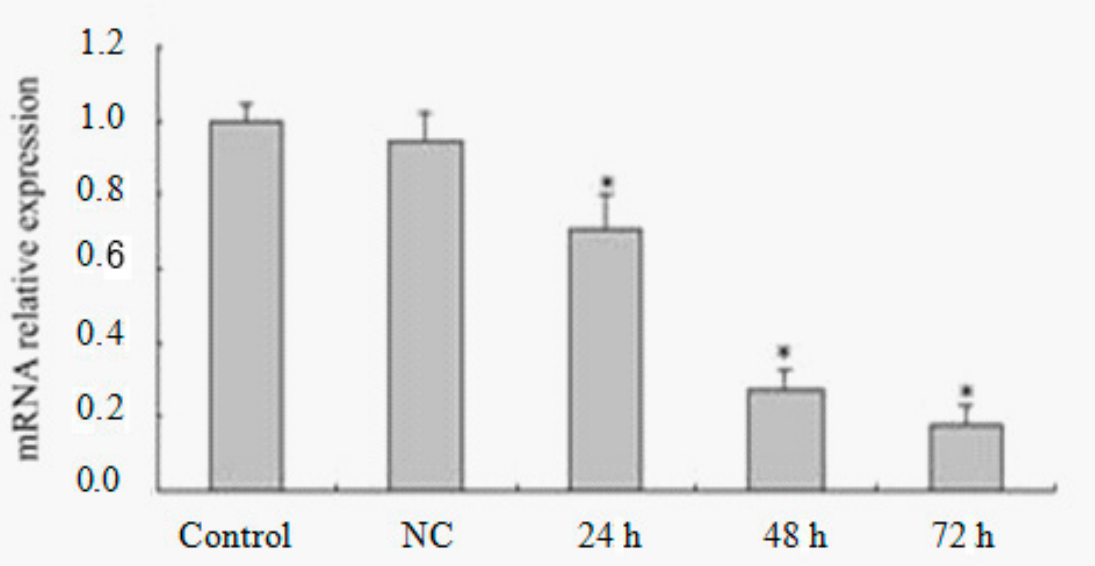

Figure 6. RT-qPCR analysis of $P T H r P$ in the mammary epithelial cells. 
The Western blotting results indicated that AD-PTHrP-322 had better knockdown efficiency than untransfected cells in primary goat mammary gland epithelial cells. The results showed that in the mammary epithelial cells infected with AD-PTHrP-322 for 24, 48, and 72 $\mathrm{h}$, protein expression of $\mathrm{PTHr} P$ was downregulated progressively compared to untransfected cells (Figure 7).

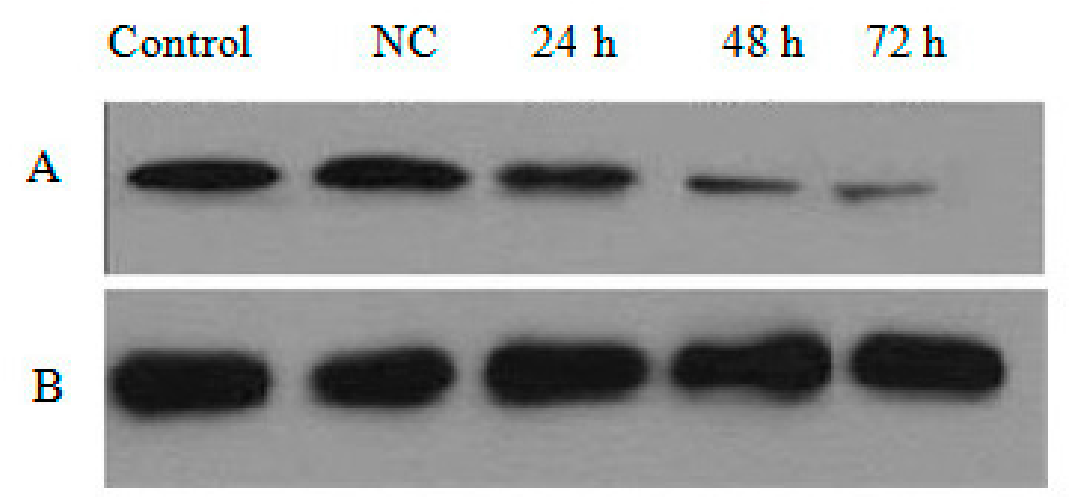

Figure 7. Analysis of interference efficiency of AD-PTHrP-322 by Western blotting. A. PTHrP. B. $\beta$-actin.

These results suggest that AD-PTHrP-322 had ability to interfere with PTHrP gene expression both at the mRNA and at the protein level.

\section{DISCUSSION}

RNAi is a form of post-transcriptional gene silencing mediated by siRNAs. It is highly specific, highly efficient, and heritable, and is an effective method for studying gene function (Xia et al., 2002; Sashital and Doudna, 2010). Recently, many groups have reported the use of synthesized oligonucleotides or siRNA-encoding plasmids to induce RNAi in mammalian cells by transfection. However, this approach is still limited in its application, especially for achieving long-term gene silencing in vivo (Hao al., 2005). To circumvent this problem, lentiviral-delivered RNAi has been developed. For our study, we chose replication-defective adenovirus vectors lacking E1 and E3 regions as these viruses can not replicate in mammalian cells. The packaging of the virus can be achieved because the HEK-293 cell line can provide the virus E1 and E3 components. Here, we designed and built an adenovirus vector encoding shRNA that targets the goat PTHrP gene. This shRNA can fold in cells to form a 19-bp hairpin loop. This structure can be processed into siRNA by the enzyme Dicer. The siRNA guide chain helicase, ATP, and multiple proteins form the RNA-induced silencing complex, which can identify and pair with the target mRNA by base pairing. Then, the siRNA binds to and digests the 3'-UTR of the target mRNA to silence gene expression (Kleinhammer et al., 2010).

Over the years, many approaches have been developed for the generation of recombinant adenoviruses. These can be divided into 2 basic categories: direct plasmid construction of the recombinant adenovirus genome (Munz and Young, 1991; Miyake et al., 1996; Anderson et al., 2000; Gao et al., 2003; McVey et al., 2003) or indirect construction in vivo (Chartier et al., 1996; Hardy et al., 1997; He et al., 1998; Nakano et al., 2005; Feng et al., 2008). The 
former has been proven to be unfeasible. The latter is limited by several factors, including the low efficiency and difficulty of screening homologous recombination, the need for timeconsuming plasmid purification, and frequent contamination by wild-type adenoviruses (Anderson et al., 2000). In order to increase the efficiency of recombination and screening, the Block-iT shRNA interference system was used in this study. It includes an adenovirus shuttle vector $\mathrm{pENTR/CMV-GFP/U6} \mathrm{and} \mathrm{an} \mathrm{adenovirus} \mathrm{backbone} \mathrm{vector} \mathrm{pAD} / \mathrm{PL}-\mathrm{DEST}$. The former contains a kanamycin-resistance gene. The latter contains the ampicillin- and chloromycetinresistance genes. Furthermore, pAD/PL-DEST contains a negative selection $c c d B$ gene.

Here, we used a simple and efficient method for the construction and identification of recombinant adenoviruses. First, shRNAs were cloned into double-digested pENTR/CMVGFP/U6, and then the optimal shRNA was selected. Secondly, pENTR/CMV-GFP/U6-shRNA and the adenovirus backbone vector pAD/PL-DEST were genetically restructured by homologous recombination. Third, the recombinant products were transformed into TOP10 recipient cells and selected by negative selection using the $c c d B$ gene and by positive selection using ampicillin and chloromycetin. Finally, the recombinant adenoviral plasmid was cleaved with PacI and then transfected into HEK-293 cells to package the recombinant adenovirus.

Since RNAi silences gene expression post-transcriptionally, we used qRT-PCR and Western blotting to detect changes in $P T H r P$ mRNA and protein expression. The results showed that the expression of PTHrP in mammary epithelial cells was downregulated progressively after the cells were infected by AD-PTHrP-322 for 24, 48, and $72 \mathrm{~h}$, However, we know that this interference effect is only short-term as the shRNA is not integrated into the host cell genome (Paddison et al., 2002). A large number of studies have reported that the interference effect of siRNA lasts for approximately 1-2 weeks (Du et al., 2006). Xiong and Wang (2009) using shRNA to silence the $M K$ gene in BxPC-3 cells, found that interference was most efficient 3-5 days postinfection and that the gene expression levels returned to normal by the 9th day postinfection. The time-point of the most efficient interference was $72 \mathrm{~h}$ postinfection in our study. In summary, our results suggest that AD-PTHrP-322 significantly interferes with expression of $\mathrm{PTHrP}$ and that it can be used for further functional studies of the PTHrP gene.

\section{ACKNOWLEDGMENTS}

Research supported by the Special Fund for Agro-Scientific Research in the Public Interest (\#201103038) and the Fundamental Research Funds for the Central Universities (\#Z109021002).

\section{REFERENCES}

Anderson RD, Haskell RE, Xia H, Roessler BJ, et al. (2000). A simple method for the rapid generation of recombinant adenovirus vectors. Gene Ther. 7: 1034-1038.

Barik S (2005). Silence of the transcripts: RNA interference in medicine. J. Mol. Med. 83: 764-773.

Barlet JP, Champredon C, Coxam V, Davicco MJ, et al. (1992). Parathyroid hormone-related peptide might stimulate calcium secretion into the milk of goats. J. Endocrinol. 132: 353-359.

Chartier C, Degryse E, Gantzer M, Dieterle A, et al. (1996). Efficient generation of recombinant adenovirus vectors by homologous recombination in Escherichia coli. J. Virol. 70: 4805-4810.

Du YF, Xing YF, Zeng FQ, Lu P, et al. (2006). The inhibitory effects of siRNA expression vector on CXCR4 expression in prostate carcinoma cell lines. J. Nanjing Med. Univ. 20: 104-108. 
Feng Z, Jiang P, Wang X, Li Y, et al. (2008). Adenovirus-mediated shRNA interference against porcine circovirus type 2 replication both in vitro and in vivo. Antiviral Res. 77: 186-194.

Gaither A and Iourgenko V (2007). RNA interference technologies and their use in cancer research. Curr. Opin. Oncol. 19: 50-54.

Gao G, Zhou X, Alvira MR, Tran P, et al. (2003). High throughput creation of recombinant adenovirus vectors by direct cloning, green-white selection and I-Sce I-mediated rescue of circular adenovirus plasmids in 293 cells. Gene Ther. 10: 1926-1930.

Hao DL, Liu CM, Dong WJ, Gong H, et al. (2005). Knockdown of human p53 gene expression in 293-T cells by retroviral vector-mediated short hairpin RNA. Acta Biochim. Biophys. Sin. 37: 779-783.

Hardy S, Kitamura M, Harris-Stansil T, Dai Y, et al. (1997). Construction of adenovirus vectors through Cre-lox recombination. J. Virol. 71: 1842-1849.

He TC, Zhou S, da Costa LT, Yu J, et al. (1998). A simplified system for generating recombinant adenoviruses. Proc. Natl. Acad. Sci. U. S. A. 95: 2509-2514.

Hens JR and Wysolmerski JJ (2005). Key stages of mammary gland development: molecular mechanisms involved in the formation of the embryonic mammary gland. Breast Cancer Res. 7: 220-224.

Hens JR, Dann P, Zhang JP, Harris S, et al. (2007). BMP4 and PTHrP interact to stimulate ductal outgrowth during embryonic mammary development and to inhibit hair follicle induction. Development 134: 1221-1230.

Hildreth BE, III, Werbeck JL, Thudi NK, Deng X, et al. (2010). PTHrP 1-141 and 1-86 increase in vitro bone formation. J. Surg. Res. 162: e9-17.

Huai $\mathrm{N}$ and Ma XM (2009). RNA interference technology and neoplasm. Inner Mongolia Med. J. 05-026.

Kleinhammer A, Wurst W and Kuhn R (2010). Gene knockdown in the mouse through RNAi. Methods Enzymol. 477: $387-414$.

Kremer R, Li J, Camirand A and Karaplis AC (2011). Parathyroid hormone related protein $(P T H r P)$ in tumor progression. Adv. Exp. Med. Biol. 720: 145-160.

Lozano D, de Castro LF, Dapia S, Andrade-Zapata I, et al. (2009). Role of parathyroid hormone-related protein in the decreased osteoblast function in diabetes-related osteopenia. Endocrinology 150: 2027-2035.

McVey D, Zuber M, Brough DE and Kovesdi I (2003). Adenovirus vector library: an approach to the discovery of gene and protein function. J. Gen. Virol. 84: 3417-3422.

Miyake S, Makimura M, Kanegae Y, Harada S, et al. (1996). Efficient generation of recombinant adenoviruses using adenovirus DNA-terminal protein complex and a cosmid bearing the full-length virus genome. Proc. Natl. Acad. Sci. U. S. A. 93: 1320-1324.

Munz PL and Young CS (1991). End-joining of DNA fragments in adenovirus transfection of human cells. Virology 183: 160-169.

Nakano M, Odaka K, Takahashi Y, Ishimura M, et al. (2005). Production of viral vectors using recombinase-mediated cassette exchange. Nucleic Acids Res. 33: e76.

Onda K, Yamaguchi M, Ohashi M, Sato R, et al. (2010). Modification of the analysis of parathyroid hormone-related protein in milk and concentrations of this protein in commercial milk and milk products in Japan. J. Dairy Sci. 93 : 1861-1867.

Paddison PJ, Caudy AA, Bernstein E, Hannon GJ, et al. (2002). Short hairpin RNAs (shRNAs) induce sequence-specific silencing in mammalian cells. Genes Dev. 16: 948-958.

Philbrick WM, Wysolmerski JJ, Galbraith S, Holt E, et al. (1996). Defining the roles of parathyroid hormone-related protein in normal physiology. Physiol. Rev. 76: 127-173.

Sashital DG and Doudna JA (2010). Structural insights into RNA interference. Curr. Opin. Struct. Biol. 20: 90-97.

VanHouten JN, Dann P, Stewart AF, Watson CJ, et al. (2003). Mammary-specific deletion of parathyroid hormone-related protein preserves bone mass during lactation. J. Clin. Invest. 112: 1429-1436.

Xia H, Mao Q, Paulson HL and Davidson BL (2002). siRNA-mediated gene silencing in vitro and in vivo. Nat. Biotechnol. 20: 1006-1010.

Xiong MY and Wang KZ (2009). Recombinant adenovirus-mediated shRNA silencing of midkine gene in BxPC-3 cells. J. Nanjing Med. Univ. 23: 132-136. 